\title{
Interstitial myofibroblasts: predictors of progression in membranous nephropathy
}

\author{
I S D Roberts, C Burrows, J H Shanks, M Venning, L J McWilliam
}

\begin{abstract}
Aims-To determine the role of interstitial myofibroblasts in the progression of membranous nephropathy; and to assess the predictive value of quantifying myofibroblasts in determining long term renal outcome.
\end{abstract}

Methods-All cases of membranous nephropathy, diagnosed by renal biopsy at University Hospital of South Manchester between 1984 and 1987, were studied retrospectively. The biopsy specimens (n = 26) were reviewed and analysed morphometrically to measure interstitial volume as a proportion of the total volume of renal cortex, and numbers of interstitial myofibroblasts (cells positive for a-smooth muscle actin within the interstitium). Clinical data, with a follow up of seven to eight years, was available for 24 patients, and renal outcome was correlated with pathological changes in the initial diagnostic biopsy specimen.

Results-The number of myofibroblasts and interstitial volume were inversely correlated with creatinine clearance at the initial biopsy, and at the end of follow up. Percentage sclerosed glomeruli or stage of glomerular disease, assessed by electron microscopy, did not correlate with renal function at initial biopsy or during follow up. The number of myofibroblasts, but not interstitial volume, correlated with severity of proteinuria at initial biopsy. Of 15 biopsy specimens showing no or mild interstitial fibrosis, four showed a notable increase in the number of interstitial myofibroblasts. All of these patients developed chronic renal failure, compared with three of 11 patients whose specimens showed no or a mild increase in myofibroblast numbers.

Conclusions-Interstitial myofibroblasts play a role in the development of interstitial fibrosis and progressive renal failure in membranous nephropathy. Increased numbers of myofibroblasts in biopsy specimens showing only mild fibrosis may predict subsequent chronic renal failure. (F Clin Pathol 1997;50:123-127)

Keywords: membranous nephropathy; myofibroblasts; fibrosis.

Correspondence to: Dr I S D Roberts, Department of Pathological Sciences, The University of Manchester, Oxford Road, Manchester M13 9PT

Accepted for publication 5 November 1996

Membranous nephropathy is the commonest cause of adult nephrotic syndrome in the UK. In most patients, membranous nephropathy is idiopathic; causes of secondary membranous nephropathy include systemic lupus erythematosus, drugs, tumours, and certain infections. The outcome of membranous nephropathy is highly variable; only a minority of adults with idiopathic membranous nephropathy achieve complete remission and 6-46\% develop progressive renal failure over time. ${ }^{1-6}$ Clinical predictors of progressive disease in idiopathic membranous nephropathy are older age, heavy proteinuria, and impaired renal function at presentation. ${ }^{357}$ The characteristic pathological change in membranous nephropathy is thickening of the glomerular capillary walls, associated with subepithelial immune deposits, but there are also changes within the tubulointerstitial compartment: a chronic inflammatory cell infiltrate, tubular atrophy, and fibrosis are frequent findings. The pathogenesis of these interstitial changes is poorly understood..$^{8-10}$ Several studies have shown that, as in other primary glomerular diseases, long term renal outcome correlates more closely with tubulointerstitial changes than the severity of the glomerular pathology. ${ }^{2411}$ At present, there is no treatment for membranous nephropathy which effectively prevents progression to chronic renal failure..$^{3-5}$ Interstitial inflammation and fibrosis are potential targets for treatment and there is, therefore, a need for a better understanding of the mechanisms underlying these changes, and also for ways to identify early and potentially reversible stages in the development of interstitial fibrosis.

As in other tissues, such as healing wounds, an early event in renal fibrosis is the proliferation of cells with a myofibroblast phenotype. ${ }^{12-14}$ These cells are recognised under light microscopy by their morphology and the expression of $\alpha$-smooth muscle actin $(\alpha-S M A) .{ }^{15}{ }^{16}$ In the normal kidney, expression of $\alpha-S M A$ is seen as peritubular staining within the cytoplasmic processes of sparse interstitial myofibroblasts (IMF). In renal fibrosis, there is a notable increase in $\alpha$-SMA staining, indicating that IMF play a central role in the fibrotic process. ${ }^{14}$ Recent studies reported that this increase in IMF is predictive of progressive disease in an animal model of glomerulonephritis, ${ }^{17}$ and in human IgA nephropathy. ${ }^{18} 19$ In this study, we investigated the role of IMF in progression of membranous nephropathy and determined the value of quantifying IMF in predicting long term outcome.

\section{Methods}

Archival biopsy material was obtained from the Department of Histopathology, The University 
Hospital of South Manchester. All biopsy specimens on which an initial diagnosis of membranous nephropathy had been made between 1984 and 1987 were reviewed, and analysed morphometrically, without knowledge of the clinical data.

The following features were sought by one of us (ISDR) on haematoxylin and eosin, and methenamine silver-stained sections: percentage of total glomeruli which were globally sclerosed; extent of tubular atrophy, graded $0-3(0$, absent; $1,1-25 \%$ of tubules; $2,26-50 \%$ of tubules; and $3,>50 \%$ of tubules); absence or presence of a chronic inflammatory cell infiltrate within the interstitium $(>25 \%$ of the interstitium); and numbers of IMF which were assessed semiquantitatively and graded 0-3. Stage of glomerular disease was assessed by review of electron micrographs.

Interstitial volume was measured as a proportion of the total renal cortex using a Chalkley graticule (Graticules Ltd, Tonbridge, UK) which contains 25 random dots. The entire cortical area was counted in the original methenamine silver-stained sections from each biopsy specimen by one of us (JHS). Glomeruli and large arteries were excluded. Interstitial fibrosis was graded separately from zero to three ( 0 , absent; $1,1-25 \%$ of specimen area; 2 , $26-50 \%$ of specimen area; $3,>50 \%$ of specimen area). Interstitial oedema was not present in any of the specimens.

Interstitial myofibroblasts were identified by their morphology and positive staining with anti- $\alpha$-SMA (Dako, High Wycombe, UK). Paraffin wax sections were stained immunohistochemically using a standard immunoperoxidase method. Numbers of IMF were counted by one of us (CB) using a squared graticule at high magnification $(\times 40)$. Counts are expressed as mean number/field; a single field had an area of $0.0156 \mathrm{~mm}^{2}$. Fields containing glomeruli and large vessels were excluded. As most of the $\alpha$-SMA positivity was within cytoplasmic processes, IMF were counted only when $\alpha$-SMA staining surrounded a nucleus. When very large numbers of IMF were present, the counts became unreliable and these cases were then expressed as $>10$ IMF/field.

The following clinical parameters were obtained from review of patient case notes: age, sex, duration of symptoms, serum creatinine and creatinine clearance at the time of initial biopsy and at the end of follow up, 24 hour urine protein, and blood pressure at presentation. Patients were followed for seven to eight years, or until death, or dialysis. Patients were divided into four groups according to outcome at the end of the follow up period: normal renal function (serum creatinine $<120 \mu \mathrm{mol} / \mathrm{l}$; creatinine clearance $>60 \mathrm{ml} / \mathrm{min}$ ); mild renal failure (serum creatinine 120-250 $\mu \mathrm{mol} / \mathrm{l}$; creatinine clearance $30-60 \mathrm{ml} / \mathrm{min}$ ); severe renal failure (serum creatinine $250-600 \mu \mathrm{mol} / \mathrm{l}$; creatinine clearance $12.5-30 \mathrm{ml} / \mathrm{min}$ ); end stage disease (dialysis; or serum creatinine $>600 \mu \mathrm{mol} / \mathrm{l}$; creatinine clearance $<12.5 \mathrm{ml} / \mathrm{min}$ ).

Results were analysed statistically using SPSS for Windows software. Histological and clinical parameters were analysed for normal distribution. Serum creatinine and creatinine clearance were analysed at biopsy and at the end of follow up using a Pearson's correlation (two-tailed) test. The association of histological features with clinical outcome was also analysed using the Fisher exact test. A p value of $<0.05$ was considered significant.

\section{Results}

CLINICAL OBSERVATIONS

Table 1 summarises the clinical and histological data for each patient. Clinical data at the time of biopsy was available for 24 of the 26 patients, and follow up data for 23. Review of case notes revealed that three patients (cases 22-24) had a secondary (drug-induced) membranous nephropathy. These patients were excluded from analysis of correlations with renal function at follow up. At the end of the follow up period, of the 20 patients with idiopathic membranous nephropathy, six had normal renal function, two showed mild renal failure, seven had end stage disease, and five had died-two with severe renal failure and three with end stage disease.

Creatinine clearance at the end of follow up showed a significant correlation with age $(p<0.0001)$, and creatinine clearance $(\mathrm{p}<0.0001)$ at presentation, but not with severity of proteinuria, blood pressure, or serum creatinine at presentation. The three patients with drug-induced membranous nephropathy made a full recovery (normal renal function, no proteinuria) following withdrawal of the drug.

\section{HISTOLOGICAL FEATURES}

In five of the 26 biopsy specimens, immunohistochemistry for $\alpha$-SMA revealed delicate staining of sparse peritubular IMF, as is seen in normal kidney (fig 1A). In 21 specimens, there was an increase in the number of IMF; staining for $\alpha$-SMA showed more widespread peritubular positivity (fig 1B). Staining was particularly prominent around glomeruli and in areas of fibrosis, around atrophic tubules. The range of the myofibroblast count was 0.1 to $>10 /$ field, median 3.59. There was a close correlation between numbers of IMF/field and numbers of IMF assessed semiquantitatively; all biopsy specimens containing $>4 \mathrm{IMF} /$ field were given a semiquantitative grade of 3 .

Eighteen of 24 biopsy specimens showed no or mild interstitial fibrosis, and 17 of these were graded 0 or 1 for tubular atrophy. The range of the Chalkley count of interstitial volume was 1.89-12.66, median 5.1. There was a close correlation between interstitial volume and severity of interstitial fibrosis and tubular atrophy (assessed semiquantitatively); all specimens with a Chalkley count of $<7$ were graded 0 or 1 (no or mild fibrosis and tubular atrophy). Interstitial volume did not correlate significantly with the number of IMF ( $r=0.3625$, $\mathrm{p}=0.069$ ).

Numbers of IMF correlated significantly with serum creatinine at biopsy $(p=0.022)$ and inversely with creatinine clearance $(p=0.001)$. Interstitial volume also correlated significantly with serum creatinine $(p=0.0001$ 

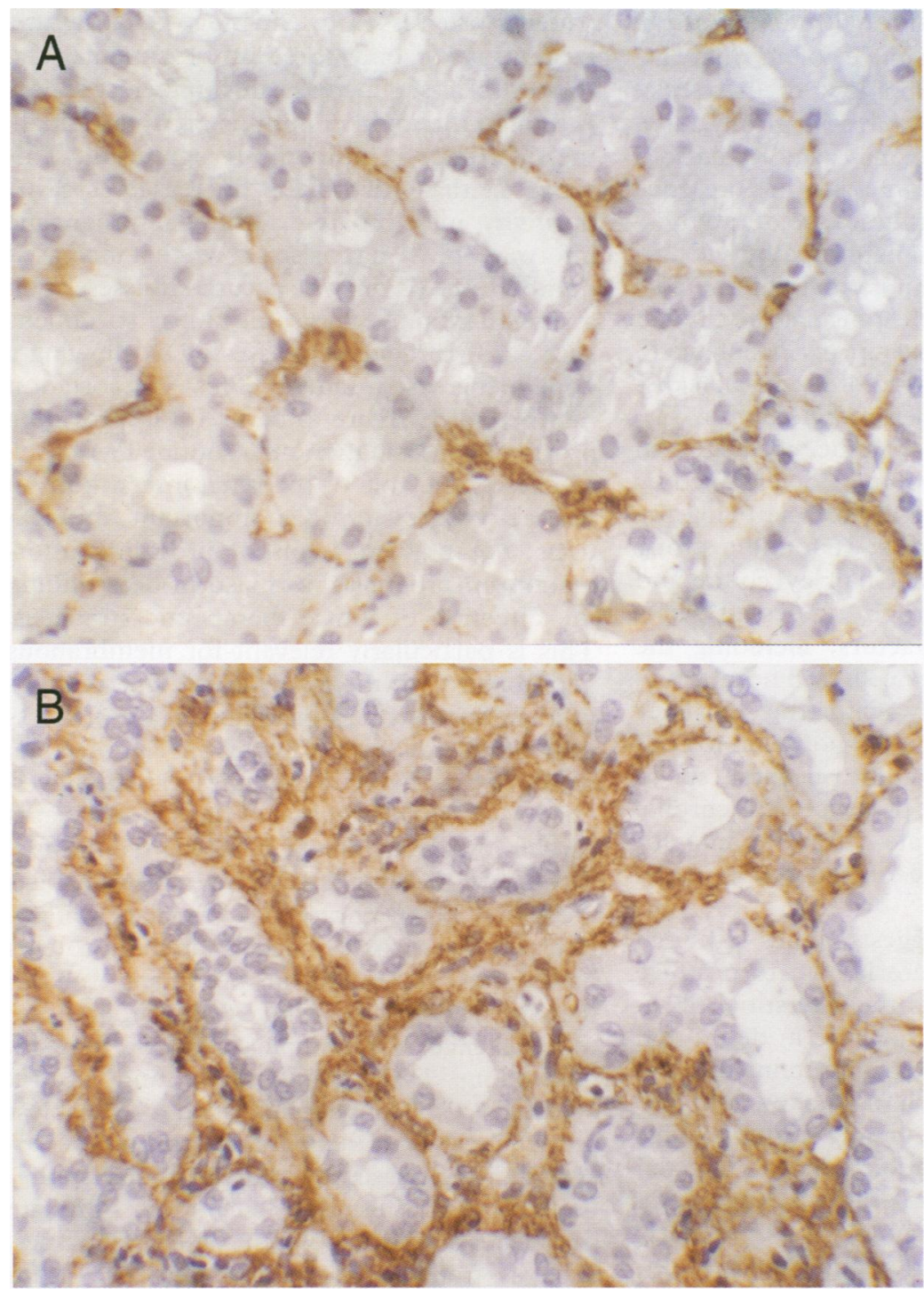

Figure 1 Staining for a-SMA showing delicate peritubular positivity in a biopsy specimen with normal numbers of interstitial myofibroblasts $(A)$, and a moderate increase in peritubular positivity in a specimen containing increased numbers of myofibroblasts (B) (original magnification, $\times 25$ ). and inversely with creatinine clearance $(p=0.001)$. Both numbers of IMF and interstitial volume were inversely correlated with creatinine clearance at the end of follow up ( $p=0.03$ and 0.01 respectively). The percentage sclerosed glomeruli, the stage of glomerular disease assessed by electron microscopy, and the presence or absence of a chronic inflammatory cell infiltrate within the interstitium were not significantly correlated with renal function at biopsy or follow up. Numbers of IMF, but not interstitial volume, correlated with severity of proteinuria at presentation $(p=0.02)$.

Of the biopsy specimens from patients with idiopathic membranous nephropathy, 15 showed no or mild interstitial fibrosis (grade 0 to 1 ; Chalkley count $<7$ ). Of these, four showed a notable increase in numbers of myofibroblasts (grade $3 ;>4 /$ field). At follow up, three of these patients had died-two with end stage disease and one with severe renal failure-and one was alive on dialysis. Of the 11 patients whose specimens showed a lesser increase in myofibroblasts, only three had progressed to renal failure (Fisher test, $\mathrm{p}=0.05$ ).

Of the biopsy specimens from the three patients with secondary membranous nephropathy, one showed moderate (grade 2) and two showed mild (grade 1) interstitial fibrosis. All showed a notable increase in myofibroblast numbers (table 1).

\section{Discussion}

It is now accepted that changes within the interstitial compartment have a major impact on long term renal outcome in many primary glomerular diseases. The presence of interstitial fibrosis on renal biopsy specimens is predictive of progression to renal failure in membranous nephropathy, as in several other primary glomerulopathies. ${ }^{2}{ }^{11}$ 20-22 Although established interstitial fibrosis is usually

Table 1 Summary of histological and clinical data

\begin{tabular}{|c|c|c|c|c|c|c|c|c|c|c|c|c|}
\hline $\begin{array}{l}\text { Patient/ } \\
\text { Sex }\end{array}$ & $\begin{array}{l}\text { Age } \\
\text { (years) }\end{array}$ & $\begin{array}{l}\text { Urine } \\
\text { protein }\end{array}$ & $\begin{array}{l}\text { sCrat } \\
\text { biopsy }\end{array}$ & $\begin{array}{l}\text { CrCl at } \\
\text { biopsy }\end{array}$ & $\begin{array}{l}\text { IMF } \\
\text { (grade) }\end{array}$ & IV (grade) & $\begin{array}{l}\text { Tubular } \\
\text { atrophy }\end{array}$ & $\begin{array}{l}\text { Chronic } \\
\text { inflammation }\end{array}$ & $\begin{array}{l}\text { Glomerular } \\
\text { sclerosis (\%) }\end{array}$ & Stage & $\begin{array}{l}\mathrm{CrCl} \\
\text { (year) }\end{array}$ & $\begin{array}{l}\text { Renal } \\
\text { status }\end{array}$ \\
\hline $1 \mathrm{~F}$ & 64 & 12.6 & 190 & 35 & $8.6(3)$ & $5.1(1)$ & 1 & + & 11 & III & $4(5)$ & $\mathrm{ESD}^{\star}$ \\
\hline $2 M$ & 43 & 2.0 & 90 & 150 & $3.2(2)$ & $5.1(1)$ & 1 & - & 9 & III & $88(4)$ & $\mathbf{N}$ \\
\hline $3 M$ & 63 & 18.4 & 130 & 62 & $8.8(3)$ & $4.8(1)$ & 1 & + & 0 & II & $15(2)$ & $\mathrm{SRF}^{\star}$ \\
\hline $4 \mathrm{M}$ & 46 & 10.1 & 80 & 137 & $0.8(1)$ & $4.7(1)$ & 1 & - & 0 & II & $22(2)$ & ESD \\
\hline $5 \mathrm{~F}$ & 41 & 1.0 & 110 & 68 & $0.9(1)$ & $2.7(1)$ & 1 & - & 11 & III & $74(7)$ & $\mathbf{N}$ \\
\hline $6 \mathrm{M}$ & 47 & 4.7 & 70 & 160 & $2.7(2)$ & $3.5(1)$ & 0 & - & 0 & II & $92(7)$ & $\mathrm{N}$ \\
\hline $7 \mathrm{M}$ & 32 & 5.6 & 60 & 185 & 0.1 (1) & $1.9(0)$ & 1 & - & 7 & III & $166(7)$ & $\mathbf{N}$ \\
\hline $8 M$ & 16 & 8.4 & 70 & 77 & $2.4(1)$ & $1.9(0)$ & 0 & - & 0 & III & $95(8)$ & $\mathbf{N}$ \\
\hline $9 \mathrm{~F}$ & 65 & 4.7 & 60 & 97 & $1.1(1)$ & $5.1(1)$ & 3 & + & 21 & II & $41(8)$ & MRF \\
\hline $10 \mathrm{~F}$ & 54 & 1.8 & 90 & 81 & $3.6(3)$ & $5.6(1)$ & 1 & + & 6 & III & $35(8)$ & MRF \\
\hline $11 \mathrm{M}$ & 67 & 11.7 & 120 & 77 & $2.9(2)$ & $6.9(1)$ & 1 & + & 9 & III & $5(4)$ & ESD \\
\hline $12 \mathrm{M}$ & 72 & 2.0 & 120 & 60 & $3.8(3)$ & $4.1(1)$ & 1 & + & 17 & I & $70(6)$ & $\mathbf{N}$ \\
\hline $13 \mathrm{M}$ & & 14.0 & 110 & 99 & $7.8(3)$ & 4.8 (1) & 0 & - & 0 & II & $5(4)$ & ESD \\
\hline $14 M$ & 56 & 9.0 & 160 & 78 & $2.9(2)$ & $6.1(1)$ & 0 & - & 0 & III & $6(1)$ & $\mathrm{ESD}^{\star}$ \\
\hline $15 M$ & 66 & 6.0 & 190 & 42 & $8.5(3)$ & $5.4(1)$ & 1 & - & 0 & IV & $7(2)$ & $\mathrm{ESD}^{\star}$ \\
\hline $16 \mathrm{~F}$ & 73 & 5.6 & 310 & 22 & $>10(3)$ & $11.2(2)$ & 2 & - & 0 & $t$ & $10(3)$ & $\mathrm{ESD}^{\star}$ \\
\hline $17 \mathrm{M}$ & 61 & 11.7 & 390 & 36 & $8.0(3)$ & $8.1(2)$ & 2 & + & 9 & III & $26(4)$ & $\mathrm{SRF}^{\star}$ \\
\hline $18 \mathrm{M}$ & 55 & 6.3 & 110 & 17 & $2.6(2)$ & $12.7(3)$ & 3 & + & 50 & IV & $5(4)$ & ESD \\
\hline $19 M$ & 61 & 2.6 & 160 & 76 & $2.8(2)$ & $7.3(2)$ & 2 & + & 25 & III & $11(8)$ & ESD \\
\hline $20 \mathrm{~F}$ & 66 & 2.4 & 670 & 47 & $6.3(3)$ & $11.5(2)$ & 2 & + & 18 & III & $t$ & $t$ \\
\hline $21 \mathrm{~F}$ & 55 & 10.0 & 670 & 5 & $>10$ (3) & $11.7(2)$ & 3 & + & 17 & II & $\ddagger$ & ESD \\
\hline $22 \mathrm{~F}$ & 63 & 6.0 & 170 & 35 & $6.4(3)$ & $8.9(2)$ & 2 & + & 14 & II & $124(4)$ & $\mathrm{N}$ \\
\hline $23 \mathrm{~F}$ & 54 & 0.5 & 90 & 80 & $6.7(3)$ & 4.5 (1) & 1 & - & 0 & III & $93(2)$ & $\mathrm{N}$ \\
\hline $24 \mathrm{~F}$ & 68 & 4.3 & 130 & 65 & $>10$ (3) & $3.5(1)$ & 1 & - & 12 & III & $100(4)$ & $\mathbf{N}$ \\
\hline
\end{tabular}

$\mathrm{sCr}=$ serum creatinine; $\mathrm{CrCl}=$ creatinine clearance; $\mathrm{IV}=$ interstitial volume by Chalkley count (followed by grade of interstitial fibrosis); $\mathrm{N}=$ normal; $\mathrm{MRF}=$ mild renal failure; SRF = severe renal failure; ESD = end stage disease; *died; †data not available; łdialysis dependent one month following biopsy; patients $22-24$ have secondary membranous nephropathy. 
associated with impaired renal function at the time of biopsy, there is some evidence that earlier events in the development of interstitial fibrosis can be identified histologically, at a stage when renal function may be normal. Both increased interstitial volume and the presence of a mononuclear cell infiltrate are of prognostic value in membranous nephropathy. ${ }^{11}{ }^{20} \mathrm{In}$ our biopsy specimens, interstitial volume correlated with creatinine clearance at the time of biopsy and after a follow up period of up to eight years, unlike the stage of glomerular disease or percentage of sclerosed glomeruli. The proportional interstitial volume, measured in this study by Chalkley count, is determined by a number of factors, the major one being the extent of interstitial fibrosis; we found a strong correlation between the Chalkley count and fibrosis and tubular atrophy (assessed semiquantitatively). Other factors, including the presence of inflammatory cells and numbers of IMF, correlated weakly with the Chalkley count. Interstitial oedema did not seem to contribute to interstitial volume in these specimens. In this study, the presence of a chronic inflammatory cell infiltrate within the interstitium was not predictive of progressive renal failure.

Recent evidence suggests that, although tubular epithelial cells are known to produce matrix components, it is the interstitial fibroblasts which play the major role in deposition of collagen and other matrix proteins in renal fibrosis. ${ }^{23}{ }^{24}$ These stromal cells show a myofibroblastic phenotype, staining positively for $\alpha$-SMA. Their proliferation can be detected before there is significant fibrosis, suggesting that they may be potentially used as early markers of progressive disease. However, unlike wound healing in the skin, the interstitial cells retain their myofibroblast phenotype following matrix deposition. Thus, increased $\alpha$-SMA positivity persists in the later stages of renal fibrosis. ${ }^{16}$ Two recent studies have investigated the potential role of IMF in progression of IgA nephropathy. ${ }^{18}{ }^{19}$ Both showed a correlation between numbers of IMF and renal function at the time of biopsy and at follow up. The number of IMF had a similar predictive value of long term outcome to that of interstitial volume. Here, we have presented similar findings in 24 patients with membranous nephropathy-numbers of IMF correlated closely with renal function at biopsy and with outcome. Interestingly, we have also shown a correlation between number of IMF and severity of proteinuria, supporting the proposal that proteins within the glomerular filtrate are the initial trigger to the tubulointerstitial changes. $^{24} 25$

As the number of $\alpha$-SMA positive IMF increases from an early stage in the development of interstitial fibrosis, their identification may potentially be used to predict progressive disease before there is extensive fibrosis. Of the 15 biopsy specimens from the patients with idiopathic membranous nephropathy which showed little or no interstitial fibrosis or tubular atrophy, four showed a notable increase in numbers of peritubular IMF. All of these patients progressed to end stage renal failure, in contrast to only three of 11 patients who showed a lesser increase in numbers of IMF. Although the numbers in this group are small, the difference in outcome is significant. This suggests that identifying increased $\alpha$-SMA positivity may provide additional prognostic information. We have also shown that increased numbers of IMF may be accurately assessed on a semiquantitative basis; all biopsy specimens with $>4 \mathrm{IMF} /$ field were assessed as showing a notable increase (grade 3 ). This is important if numbers of IMF are to be useful in providing prognostic information on a routine basis.

The central role of the renal interstitium in disease progression has important implications, not only in terms of providing information on prognosis, but also in determining the direction of future therapeutic strategies. This is particularly relevant for membranous nephropathy as the many treatment regimes used so far have had little impact on progression of the disease. What is clearly needed, before more directed therapies can be developed, is an understanding of the cellular events early in the development of interstitial fibrosis. The events that regulate the interstitial changes are slowly being unravelled. Vascular obliteration and direct toxic effects of filtered proteins have been implicated in producing tubular damage. ${ }^{9}$ There is growing evidence to indicate the existence of a complex cytokine network controlling IMF proliferation and matrix synthesis. ${ }^{10}{ }^{26-29}$ Cytokines, such as fibroblast growth factor, interleukin-1 and tumour necrosis factor- $\alpha$, released by injured tubular epithelial cells and infiltrating leucocytes, stimulate mitogenesis of fibroblasts. Other cytokines, such as transforming growth factor- $\beta$, again released by tubular cells and macrophages, switch off proliferation and stimulate production of matrix proteins. Whereas advanced interstitial fibrosis and tubular atrophy are unlikely to be reversible to any great extent, the earlier stage of IMF proliferation, without significant fibrosis, is potentially reversible. Interestingly, the three patients with secondary, drug-induced, membranous nephropathy all had an excellent long term outcome, despite all three biopsy specimens showing a notable increase in the number of IMF. As potential therapeutic agents which interrupt the production and action of fibrogenic cytokines are developed, it will become increasingly important for pathologists to identify those patients at risk of developing progressive renal fibrosis. Assessing numbers of interstitial myofibroblasts seems to be a relatively cheap and simple means of doing so.

1 Mallick NP, Short CD, Manos J. Clinical membranous nephropathy. Nephron 1983;34:209-19. 2 Ramzy MH, Cameron JS, Turner DR, Neild GH, Ogg CS, Hicks J. The long term outcome of idiopath.

3 Donadio JV, Torres VE, Velosa JA, Wagoner RD, Holley KE, Okamura $\mathrm{M}$, et al. Idiopathic membranous nephropathy: the natural history of untreated patients. Kidney In 1988;33:708-15.

4 Wehrmann M, Bohle A, Bogenschutz O, Eiselle R, Freislederer A, Ohlschlegel $\mathrm{C}$, et al. Long term prognosis of chronic idiopathic membranous glomerulonephritis. Clin Nephrol 1989;31:67-76. 
5 Honkanen E. Survival in idiopathic membranous glomerulonephritis. Clin Nephrol 1986;25:122-8.

6 Davison AM, Cameron JS, Kerr DNS, Ogg CS, Wilkinson RW. The natural history of renal function in untreated idiopathic membranous glomerulonephritis in adults. Clin Nephrol 1984;22:61-7.

7 Murphy BF, Fairley KF, Kincaid-Smith PS. Idiopathic membranous glomerulonephritis: long term follow up in 139 cases. Clin Nephrol 1988;30:175-81.

8 Ong ACM, Fine LG. Loss of glomerular function and tubulointerstitial fibrosis: cause or effect? Kidney Int 1994;45: $345-51$.

9 Fine LG, Ong ACM, Norman JT. Mechanisms of tubulointerstitial injury in progressive renal diseases. Eur $\mathcal{f}$ tubulointerstitial injury in prog

10 Kuncio GS, Neilson EG, Haverty T. Mechanisms of Kuncio GS, Neilson EG, Haverty T. Mechanism

11 Alexopoulos E, Seron D, Hartley RB, Nolasco F, Cameron JS. Immune mechanisms in idiopathic membranous nephropathy: the role of interstitial infiltrates. Am $\mathcal{F}$ Kidney Dis 1989;13:404-12.

12 Gabbiani G. The biology of the myofibroblast. Kidney Int 1992;41:530-2.

13 Johnson RJ, Floege J, Yoshimura A, Iida H, Couser WG, Alpers CE. The activated mesangial cell: an activated myofibroblast? $7 \mathrm{Am}$ Soc Nephrol 1992;2:190-7.

14 Alpers CE, Hudkins KL, Floege J, Johnson RJ. Human renal cortical interstitial cells with some features of smooth muscortical interstitial cells with some features of smooth muscle cells participate in tubulointerstitial
injury. $\mathcal{F} \mathrm{Am}$ Soc Nephrol 1994;5:201-10.

15 Skalli O, Schurch W, Seemayer T, Lagace R, Montandon D, Pittet B, Gabbiani G. Myofibroblasts from diverse pathological settings are heterogenous in their content of actin isoforms and intermediate filament proteins. Lab Invest 1989;60:275-285.

16 Sappino AP, Schurch W, Gabbiani G. Differentiation repertoire of fibroblastic cells: expression of cytoskeletal proteins as marker of phenotypic modulations. Lab Invest 1990;63: $144-61$.

17 Zhang G, Moorhead PJ, El Nahas AM. Myofibroblasts and the progression of experimental glomerulonephritis. Exp Nephrol 1995;3:308-18.
18 Goumenos DS, Brown CB, Shortland J, El Nahas AM. Myofibroblasts, predictors of progression of mesangial IgA nephropathy? Nephrol Dial Transplant 1994;9:1418-25.

19 Hewitson TD, Becker GJ. Intersitial myofibroblasts in IgA glomerulonephritis. Am $\mathcal{f}$ Nephrol 1995;15:111-17.

20 Bohle A, Mackensen-Haen S, Gise H. Significance of tubulointerstitial changes in the renal cortex for the excretory
function and concentration ability of the kidney: a function and concentration ability of the kidney: a
morphometric contribution. Am $\mathcal{F}$ Nephrol 1987;7:421-33.

21 Bohle A, Muller GA, Wehrmann M, Mackensen-Haen, Xiao J-C. Pathogenesis of chronic renal failure in the primary glomerulopathies, renal vasculopathies and chronic interstitial nephritides. Kidney Int 1996;49:S2-9.

22 Austin HA, Boumpas DT, Vaughan EM, Balow JE. Predicting renal outcomes in severe lupus nephritis: contributions of clinical and histological data. Kidney Int 1994;45:544-50.

23 Rodemann HP, Muller GA. Characterisation of human renal fibroblasts: II. In vitro growth, differentiation and collagen synthesis of fibroblasts from kidneys with interstitial fibrosis. Am 7 Kidney Dis 1991;17:684-6.

24 Eddy AA. Experimental insights into the tubulointerstitial disease accompanying primary glomerular lesions. $f \mathrm{Am}$ Soc Nephrol 1994;5:1273-87.

25 Eddy AA. Interstitial nephritis induced by protein-overload proteinuria. Am ₹ Pathol 1989;135:719-33.

26 Floege J, Grone H-J. Progression of renal failure: what is the role of cytokines? Nephrol Dial Transplant 1995;10:1575-86.

27 Ong ACM, Fine LG. Tubular derived growth factors and cytokines in the pathogenesis of tubulointerstitial fibrosis: implications for human renal disease progression. $\mathrm{Am} \mathcal{F}$ Kidney Dis 1994;23:205-9.

28 Lonnemann G, Shapiro L, Engler-Blum G, Muller GA, Koch KM, Dinarello CA. Cytokines in human renal fibrosis. I. Interleukin-1 is a paracrine growth factor for culture fibrosis-derived kidney fibroblasts. Kidney Int 1995;47: 837-44.

29 Strutz F, Neilson EG. The role of lymphocytes in the progression of interstitial disease. Kidney Int 1994;45: S106-10. 